\title{
Aspects génétiques de l'infertilité
}

\author{
B. SELE
}

Biologie de la Reproduction - CHU de Grenoble INSERM U 309 Institut Albert Bonniot

38043 Grenoble Cedex

\section{RÉSUMÉ}

La fertilité d'un couple dépend pour partie de la qualité de la gamétogenèse qui comporte les divisions méiotiques et les phénomènes de différenciation aboutissant à la production de gamètes mûrs. Ces phénomènes sont placés sous le contrôle de nombreux gènes dont la plupart restent à identifier. La fertilité féminine est liée à la fécondabilité d'une unique cellule produite au cours du cycle, alors que la fertilité masculine dépend de la fonctionnalité d'une population hétérogène de spermatozoïdes. Il existe un faisceau d'arguments scientifiques en faveur de l'absence de corrélation entre la fonctionnalité d'un spermatozoïde et le génome haploïde qu'il véhicule.

Mots-clés : gamétogenèse - cytogénétique - infertilité - fécondation.

\section{Fertilité du couple et Fécondance des gamètes}

La fertilité qualifie l'aptitude d'un couple à procréer. Elle est corrélée à de nombreuses variables telles que le taux basal de FSH chez la femme ou la numération des spermatozoïdes dans l'éjaculat chez l'homme ; d'où l'aspect quantifiable de la fertilité, qui devient une stérilité lorsqu'est atteint le degré extrême d'hypofertilité. Comme toutes les fonctions, la fertilité est placée sous le contrôle du géno- me somatique des deux individus qui forment un couple.

La fécondance, pour sa part, qualifie l'aptitude d'une cellule germinale à fusionner avec une cellule de "sexe" opposé. Il n'existe pas de degré de fécondance ni de fécondabilité : la cellule est apte ou inapte à cette fonction. Indirectement cette aptitude est aussi placée sous contrôle du génome somatique de l'individu dont la cellule est issue.

Fertilité et fécondance sont liées : en effet la fertilité de l'individu dépend pour partie de la production de gamètes fécondants ou fécondables. Cependant ces deux qualifications ne sont en rien équivalentes, car la fertilité met en jeu un organisme intégré alors que la fécondance relève de la biologie d'une cellule.

La fertilité est étroitement corrélée à la qualité de la gamétogenèse dont le produit ultime est le gamète mûr. Chez tous les mammifères le sexe mâle se distingue du sexe femelle par la permanence et le nombre élevé de gamètes produits. C'est l'ensemble des spermatozoïdes présents au sein d'un éjaculat qui doit être considéré et non une unique cellule comme dans le sexe femelle. Or la population de spermatozoïdes d'un éjaculat présente un degré extrême d'hétérogénéité.

En premier lieu l'hétérogénéité est génétique : le génome haploïde est constitué à l'issue des deux divisions de méiose c'est à dire au stade de spermatide. Or la méiose présente de fréquentes mutations géniques ou chromosomiques à l'origine de constitutions génétiques 
anormales. Dans l'espèce humaine, il est établi qu'environ $8 \%$ des spermatozoïdes d'un éjaculat comportent un génome gravement altéré.

En second lieu l'hétérogénéité est fonctionnelle. A l'issue de la spermiogenèse, c'est à dire de la maturation des spermatides en spermatozoïdes, il existe une grande variabilité en terme de vitalité (de nombreux spermatozoïdes sont morts), en terme de morphologie (de nombreux spermatozoïdes présentent des atypies) et en terme de mobilité. Or ces paramètres sont impliqués dans la fonctionnalité des spermatozoïdes, c'est à dire dans leur pouvoir fécondant. On peut donc distinguer deux souspopulations de spermatozoïdes selon qu'ils sont fécondants ou non fécondants.

\section{Génome haploïde et fécondance du spermatozoïde}

La question essentielle est celle du degré de recouvrement entre la sous-population de spermatozoïdes non fécondants et celle des spermatozoïdes à contenu génétique anormal. De la réponse à cette question dépend en effet le bien fondé de la pratique de l'ICSI, qui a pour objet l'obtention d'un oeuf fécondé à partir d'un spermatozoïde fonctionnellement défaillant.

Deux théories sont en faveur d'une corrélation entre le contenu génétique du spermatazoïde et son pouvoir fécondant ; la première ne repose sur aucun argument scientifique : elle postule que l'accès à l'ovocyte procède d'une mise en compétition des spermatozoïdes sur le plan génétique, par assimilation de ces cellules à une horde de mâles dont le plus performant s'approprierait le plus grand nombre de femelles, confèrant un avantage sélectif au génome qu'il transmet. Outre que le spermatozoïde ne peut être impliqué qu'une seule fois dans une fécondation, l'assimilation est abusive car elle confond la physiologie d'un organisme entier et celle d'une cellule. Cette théorie n'est pas très éloignée de la théorie archaïque de la préformation qui postulait l'exclusivité du génome mâle à participer à celui du zygote, l'ovocyte ne constituant qu'un réceptacle génétiquement neutre.

La seconde théorie est une interprétation peu scientifique de la présence de transcrits haploïdes dans la cellule germinale. Si cette présence est avérée [3], il n'en reste pas moins qu'il s'agit de transcrits ubiquitaires qui, selon toute vraisemblance, sont à mettre sur le compte des remaniements considérables que subit la chromatine au cours de la spermiogenèse. Certes on observe parmi eux des transcrits tout à fait spécifiques du testicule, mais la nature haploïde de ces derniers reste à démontrer, sachant qu'ils sont aussi présents dès le stade pachytène, c'est à dire à un stade diploïde, avant que les recombinaisons génétiques n'aient eu lieu. Ces transcrits spécifiques peuvent donc provenir du génome somatique des cellules souches germinales, voire des autres cellules somatiques testiculaires.

A l'inverse d'autres arguments plaident en faveur de l'absence de corrélation entre le contenu chromosomique d'un spermatozoïde et sa fonctionnalité. Ainsi on observe le même taux d'anomalies chromosomiques lorsqu'on l'évalue par le caryotype qui n'est établi que sur des spermatozoïdes fécondants, ou par l'hybridation in situ qui explore l'ensemble des spermatozoïdes vivants de l'éjaculat. En première approximation on peut donc considérer qu'il n'existe pas de corrélation entre le pouvoir fécondant d'un spermatozoïde et le génome qu'il véhicule. S'il existe une sélection des spermatozoïdes dans les voies génitales féminines, celle-ci ne concerne que la fonctionnalité de ces cellules et la fécondation reste un phénomène hautement aléatoire sur le plan génétique.

Si l'on considère enfin l'origine et la quantité de messagers présents dans l'oeuf fécondé humain, il apparaît une décroissance des messagers d'origine maternelle jusqu'à J4 ou J5, puis une apparition de messagers d'origine zygotiques, mais on n'a pas démontré la présence de messagers d'origine paternelle. Lorsqu'un effet paternel est mentionné [7] il est vraisemblablement d'origine épigénétique.

A cette absence de corrélation il existe cependant une exception notoire : c'est celle des spermatozoïdes atypiques macrocéphales au niveau desquels des polyploïdies ont été mises en évidence par hybridation in situ [11]. La pratique de l'ICSI avec de tels spermatozoïdes, d'ailleurs techniquement laborieuse, permet 
d'observer des oeufs fécondés à l'évidence triploïdes contenant un pronoyau femelle haploïde et un pronoyau mâle diploïde.

\section{L'infertilité de cause génétique ou l'infertilité héréditaire}

Si la fonctionnalité d'un spermatozoïde n'est pas à mettre en relation avec le génome qu'il porte, il n'en reste pas moins que la production de spermatozoïdes fonctionnels, c'est à dire l'ensemble de la spermatogenèse, y compris la méiose, est placée sous le contrôle du génome somatique. De très nombreux gènes sont impliqués et restent pour l'essentiel à découvrir par l'exploration génétique de l'infertilité d'origine masculine.

Il existe ainsi des situations d'infertilité masculine dont l'origine génotypique est déjà démontrée ou suspectée. Il peut s'agir de causes géniques telles que les mutations du gène CFTR, le syndrome de Kartagener ou les microdélétions du chromosome $\mathrm{Y}$ dans la région $\mathrm{AZF}$ en particulier. La mise en évidence de telles microdélétions contribue d'ailleurs à une meilleure appréciation des corrélations phénotype-génotype, car elle apporte la preuve d'une corrélation entre le phénotype " spermatogenèse altérée " et le génotype somatique " chromosome Y délété ". A l'inverse elle va à l'encontre de la thèse selon laquelle la fécondance d'un spermatozoïde serait corrélée à son propre génotype, car selon cette hypothèse, seuls les spermatozoïdes porteurs de l'Y délété devraient être défaillants, alors que les spermatozoïdes porteurs d'un chromosome X pourraient être fécondants.
Il existe aussi des infertilités en relation avec des aberrations chromosomiques telles que des translocations ou des anomalies du nombre des gonosomes au premier rang desquelles se situe le syndrome de Klinefelter. Or aujourd'hui les sujets Klinefelter qui présentent une spermatogénèse résiduelle peuvent procréer grâce à l'ICSI [9]. Mais alors se trouve posée la question de la transmission éventuelle du syndrome à leur descendance mâle. Grâce aux techniques d'hybridation in situ de sondes spécifiques des chromosomes $\mathrm{X}, \mathrm{Y}$ et d'un autosome, il est possible d'évaluer la proportion de spermatozoïdes $24 \mathrm{XY}$ responsables de cette transmission. Les résultats publiés indiquent des proportions très variables $[1,2,4,5,6,8]$ indépendamment de l'état homogène ou mosaïque du sujet (Tableau I). Par ailleurs il reste encore à explorer l'existence d'un éventuel effet interchromosomique qui pourrait être à l'origine d'anomalies autosomiques beaucoup plus invalidantes, pour la descendance. On voit donc que lorsqu'une cause génétique d'infertilité est identifiée, elle expose la descendance à un risque éventuel de perpétuer ce type d'handicap. Pour autant il n'y a pas lieu de refuser à ces sujets une prise en charge en terme de procréation artificielle, dès lors que l'information a été délivrée et qu'un consentement a été recueilli.

Pourtant la prise en charge de ces sujets, conforme à la définition du conseil génétique, ne fait pas l'unanimité [10], sur des arguments qu'il conviendrait de mieux cerner. Il est d'ailleurs surprenant que de telles réticences ne se soient pas aussi manifestées dans les cas

Tableau 1 : Contenu cytogénétique des spermatozoüdes de sujets porteurs du syndrome de Klinefelter

\begin{tabular}{lllll}
\hline $\begin{array}{l}\text { Caryotype } \\
\text { somatique }\end{array}$ & Technique & $\begin{array}{l}\text { Nb spz } \\
\text { analysés }\end{array}$ & $\begin{array}{l}\mathbf{2 4 X Y} \\
\%\end{array}$ & Réf. \\
\hline $46, \mathrm{XY} / 47, \mathrm{XXY}$ & caryotype & 543 & 0,9 & $(2)$ \\
& HIS & 3800 & 1,3 & $(8)$ \\
& FISH (X,Y,1) & 27097 & 2,09 & $(1)$ \\
$47, \mathrm{XXY}$ & & & 1,36 & $(6)$ \\
& FISH (X,Y,1) & 2206 & 25 & $(4)$ \\
& FISH (X,Y,18) & 24 & 14,6 & $(5)$ \\
& FISH (X,Y,8) & 10000 & 10 & \\
\hline
\end{tabular}


où l'infertilité est d'origine féminine. En effet il existe aussi des causes géniques à l'infertilité féminine, telles que les mutations des récepteurs des gonadotrophines, le syndrome de Kartagener, les mutations de facteurs de reconnaissance des spermatozoïdes, comme il existe des causes chromosomiques telles que certaines translocations ou anomalies des gonosomes du syndrome de Turner par exemple.

En conclusion, le nombre de paramètres qualitatifs et quantitatifs intervenant dans la fertilité est tel que tout individu peut potentiellement devenir infertile. Par ailleurs tout individu est exposé au risque de transmettre à sa descendance un ou plusieurs gènes soit simplement défavorables tels que ceux impliqués dans l'infertilité, soit gravement délétères. En somme il ne peut exister de contre-indication génétique à la procréation, que celle-ci soit naturelle ou médicalement assistée. Si la procréation sans risque génétique est devenue une exigence des temps modernes, elle est une illusion qui rejoint le désir d'immortalité.

\section{RÉFÉRENCES}

1. CHEVRET E, ROUSSEAUX S, MONTEIL M et al. Increased incidence of hyperhaploïd $24, \mathrm{XY}$ spermatozoa detected by three-colour FISH in a $46, \mathrm{XY} / 47, \mathrm{XXY}$ male. Hum Genet 1996 ; 97 : 171-175.

2. COZZI J, CHEVRET E, ROUSSEAUX $\mathrm{S}$, et al. Achievement of meiosis in XXY germ cells : study of 543 sperm karyotypes from an XY/XXY mosaic patient. Hum Genet 1994 ; 93 : 32-34.

3. ERICKSON RP. Post-meiotic gene expression. Trends Genet $1990 ; 6: 264-269$.

4. ESTOP AM, MUNNÉ S, CIEPLY KM, VANDERMARK KK, LAMB AN, FISCH H. Meiotic products of a Klinefelter $47, \mathrm{XXY}$ male as determined by sperm fluorescence in-situ hybridization analysis. Hum Reprod 1998 ; 13 : 124-127.

5. FORESTA C, GALEAZZI C, BETTELA A, STELLA M, SCANDELLARI C. High incidence of sperm sex chromosome aneuploidies in two patients with Klinefelter's syndrome. J Clin Endocrinol Metab 1998 ; 83 : 203-205.

6. GUTTENBACH M., MICHELMANN HW, HINNEY B, ENGEL W, SCHMID M. Segregation of sex chromosomes into sperm nuclei in a man with $47, \mathrm{XXY}$ Klinefelter's karyotype : a FISH analysis. Hum Genet $1997 ; 99$ : 474-477.
7. JANNY L, MENEZO Y. Evidence for a strong paternal effect on human preimplantation embryo development and blastocyst formation. Mol Reprod Dev 1994; 38 : 36-42.

8. MARTINI E, GERAEDTS JPM, LIEBAERS I et al. Constitution of semen samples from XYY and XXY males as analysed by in-situ hybridization. Hum Reprod 1996 ; 11 : 1638-1643.

9. PALERMO GD, SCHLEGEL PN, SILLS ES et al. Births after intracytoplasmic injection of sperm obtained by testicular extraction from men with nonmosaic Klinefelter's syndrome. New Engl J Med 1998 ; 338 : 588-590.

10. THIBAULT C. Certitudes et inquiétudes concernant l'ICSI. Contr Fert Sex 1998 ; 26 : 211-217.

11. YUROV YB, SAIAS MJ, VORSANOVA SG et al. Rapid chromosomal analysis of germ-line cells by FISH : an investigation of an infertile male with large-headed spermatozoa. Mol Hum Reprod 1996 ; 2 : 665-668.

\section{ABSTRACT}

\section{Genetic aspects of infertility}

B.SELE

The fertility of a couple is partially related to the quality of gametogenesis, it includes meiotic divisions as well as differenciation processes leading to the formation of mature gametes. These phenomenona are under genomic control but most of their specific genes are still unknown. Female fertility depends on the fecundability of a unique cell produced during a cycle in contrast male fertility is conditionned by the fertilizing ability of a large population of highly heterogenous sperm. Several arguments are in favor of the absence of correlation between the fertilizing ability of a sperm and its genetic content.

Key-Words : gametogenesis, cytogenetic, infertility, fertilization 